\title{
Single Nucleotide Polymorphisms in CDKAL1 Gene Are Associated with Risk of Gestational Diabetes Mellitus in Chinese Population
}

\author{
Keke Wang $(\mathbb{D}),{ }^{1}$ Qiong Chen $\left(\mathbb{D},{ }^{1,2}\right.$ Yongliang Feng, ${ }^{1}$ Hailan Yang, ${ }^{3}$ Weiwei Wu, ${ }^{1}$ Ping Zhang, \\ Ying Wang, ${ }^{1}$ Jamie Ko, ${ }^{4}$ Feng Zhao, ${ }^{1}$ Wenqiong Du, ${ }^{1}$ Feifei Yang, ${ }^{1}$ Tianbi Han, \\ Suping Wang $\mathbb{D}^{1}{ }^{1}$ and Yawei Zhang $\mathbb{D D}^{1,5,6}$ \\ ${ }^{1}$ Department of Epidemiology, Shanxi Medical University School of Public Health, Taiyuan 030001, China \\ ${ }^{2}$ Office for Cancer Prevention and Research, Affiliated Cancer Hospital of Zhengzhou University/Henan Cancer Hospital, \\ Zhengzhou 450008, China \\ ${ }^{3}$ Department of Obstetrics, the First Affiliated Hospital, Shanxi Medical University, Taiyuan 030001, China \\ ${ }^{4}$ Department of Chronic Disease Epidemiology, Yale School of Public Health, New Haven, 06520 CT, USA \\ ${ }^{5}$ Department of Surgery, Yale University School of Medicine, New Haven 06520, USA \\ ${ }^{6}$ Department of Environmental Health Sciences, Yale School of Public Health, New Haven, 06520 CT, USA
}

Correspondence should be addressed to Suping Wang; spwang88@163.com and Yawei Zhang; yawei.zhang@yale.edu

Received 22 October 2018; Revised 11 February 2019; Accepted 4 March 2019; Published 14 April 2019

Academic Editor: Daniela Foti

Copyright (C) 2019 Keke Wang et al. This is an open access article distributed under the Creative Commons Attribution License, which permits unrestricted use, distribution, and reproduction in any medium, provided the original work is properly cited.

\begin{abstract}
Gestational diabetes mellitus (GDM) is a growing public health concern for many reasons, and its etiology remains unclear. Due to the similarity of its pathophysiology with type 2 diabetes (T2DM), we evaluated the relationship between published T2DM susceptibility genes and the risk of GDM. A total of 303 SNPs from genes including IRS1, IGF2BP2, CDKAL1, GCK, TCF7L2, KCNQ1, and KCNJ11 and the risk of GDM were examined in a nested case-control study with 321 GDM cases and 316 controls. The odds ratios (ORs) and their 95\% confidence interval (95\% CI) were estimated by unconditional logistical regression as a measure of the associations between genotypes and GDM in additive, recessive, dominant, and codominant models adjusting for maternal age, maternal BMI, parity, and family history of diabetes. At the gene level, CDKAL1 was associated with GDM risk. SNPs in the CDKAL1 gene including rs4712527, rs7748720, rs9350276, and rs6938256 were associated with reduced GDM risk. However, SNPs including rs9295478, rs6935599, and rs7747752 were associated with elevated GDM risk. After adjusting for multiple comparisons, rs9295478 and rs6935599 were still significant across the additive, recessive, and codominant models; rs7748720 and rs6938256 were significant in dominant and codominant models; and rs4712527 was only significant in the codominant model. Our study provides evidence for an association between the CDKAL1 gene and risk of GDM. However, its role in the GDM pathogenesis still needs to be verified by further studies.
\end{abstract}

\section{Introduction}

Gestational diabetes mellitus (GDM) is a public health concern due to its large disease burden and its short- and long-term adverse health impact on pregnant women and their offspring including obesity, metabolic syndrome, and type 2 diabetes mellitus (T2DM) [1]. GDM affects approximately $5-17 \%$ of all pregnancies worldwide [2]. The prevalence has increased over the past 20 years, and this upward trend is expected to continue due to a rising number of overweight or obese women of childbearing age $[2,3]$. Although the pathogenesis of GDM remains unclear, it is considered as a complex disease caused by multiple factors including genetic, environment, lifestyle, and other random factors that related to reduction of insulin sensitivity and insulin resistance.

GDM shares common risk factors with T2DM, including family history of diabetes, obesity, high maternal age, 
abnormal glucose tolerance, and specific ethnicity [4]. The similarities of pathophysiology between GDM and T2DM may suggest that the genetic factors of T2DM may also be involved in the development of GDM. GDM has been suggested as precursor for T2DM by some researchers [5]. Genome-wide association studies and candidate gene studies had already identified several genes that associated with risk of T2DM, and some of them were also verified to be associated with GDM risk by candidate gene studies and one GWA study including TCF7L2, GCK, KCNJ11, KCNQ1, CDKAL1, IGF2BP2, and IRS1 genes [6]. However, there are still many genes that were previously reported to be associated with T2DM but were not associated with GDM risk. It is possible that the T2DM susceptibility genes were mostly found in GWA studies or metaanalysis normally with large sample size and weak effect, but those found in GDM studies often have small sample size resulting in insufficient statistical power to identify weak effects [7].

Current evidence suggested that the genetic polymorphisms often had different GDM risks in different ethnic populations [5,8]. Genetic polymorphisms in TCF7L2 and GCKR genes were found to be associated with GDM risk in European women, and polymorphisms in TSPAN8 were associated with increased GDM risk in African American women [9]. TCF7L2 and KCNQ1 polymorphisms were found to be associated with GDM risk in Mexican women [10]. In Asia, polymorphisms in TCF7L2, KCNQ1, KCNJ11, $I G F 2 B P 2$, and CDKAL1 genes were associated with GDM risk in the Korean population [8], while CDKAL1 and GCK gene polymorphisms were associated with GDM in Indian and Thai women [8]. However, to our knowledge, the association with GDM risk was only evaluated in IGF2BP2 and KCNQ1 genes in Chinese women [7], and other genes like TCF7L2, GCK, KCNJ11, CDKAL1, and IRS1 still need to be verified by additional studies. Thus, we examined the associations between these genes and risk of GDM using a nested case-control study design in the Taiyuan birth cohort population.

\section{Materials and Methods}

2.1. Subjects. The subjects were enrolled from an ongoing birth cohort at the First Affiliated Hospital of Shanxi Medical University during March 1, 2012, and July 30, 2014, in Taiyuan, China [11]. Eligible women included pregnant women who came to the hospital for delivery with gestational age of 20 weeks or more, who had no mental illness, and who were aged 18 years or older. Eligible women were informed of the study procedure upon their arrival at the hospital for delivery. After obtaining written consent, an in-person interview was conducted at the hospital by trained study interviewers using a standardized and structured questionnaire. The questionnaire collected information regarding demographic factors, reproductive and medical history, smoking, alcohol and tea consumption, occupational and residential histories, physical activity, and diet. Information on birth outcomes and pregnancy complications was abstracted from medical records.
2.2. Case and Control Selection. Blood glucose was tested using a $75 \mathrm{~g}$ oral glucose tolerance test (OGTT) during 24-28 weeks of gestation. Subjects were diagnosed as having GDM if they met at least one of the following criteria: (1) fasting blood glucose $>5.1 \mathrm{mmol} / \mathrm{L}$, (2) 1 -hour blood glucose $>10.0 \mathrm{mmol} / \mathrm{L}$, and/or (3) 2-hour blood glucose $>$ $8.5 \mathrm{mmol} / \mathrm{L}$. A total of 334 women had GDM (cases), and 334 control subjects who had no GDM were randomly selected through frequency matching to cases by age, month of conception, and residence. 13 cases and 18 controls were excluded due to missing genotyping per person or per SNP. Finally, 321 cases and 316 controls were included in the analysis.

2.3. Genotyping. DNA was extracted, isolated, and purified from whole blood samples according to a standard phenolchloroform extraction method. Genotyping was conducted using an Illumina GoldenGate Platform. Duplicate samples (5\%) were interspersed throughout the plates used for genotype analysis for quality control purposes. A total of 303 single-nucleotide polymorphisms (SNPs) from genes including IRS1, IGF2BP2, CDKAL1, GCK, TCF7L2, KCNQ1, and $K C N J 11$ were considered for this study. The completion rate for all SNPs was over 99\%. The Hardy-Weinberg equilibrium (HWE) was assessed in controls for each SNP using a chi-square test. SNPs with a $P$ value $>0.05$ from the chi-square test were considered to be in HWE. Of the 303 SNPs tested, 8 SNPs were not in HWE and were excluded from the final analysis (as shown in Supplementary Table 1).

2.4. Statistical Analysis. All statistical analyses were carried out using $\mathrm{R}$ statistical software version 3.3.1. The odds ratios (OR) and their 95\% confidence intervals (95\% CI) were estimated by unconditional logistical regression as a measure of the associations between genotypes and GDM adjusting for maternal age, maternal BMI, parity, and family history of diabetes. The associations were estimated in additive, dominant, codominant, and recessive models. The minimum $P(\min P)$ tests were conducted to examine an association at the gene level using "minPtest" R package [12]. The min $P$ test was based on permutation resampling and was conducted to assess the true statistical significance of the smallest $P$ trend within each gene region. Haplotype analyses were conducted for all genes in which more than 1 SNP was genotyped. Haplotype block structures were evaluated with HaploView version 4 using the method of Four Gamete Rule. Logistic regression was used to calculate the ORs of the haplotypes using the method implemented in the "haplo.ccs" package [13]. Only major haplotypes with an estimated frequency of over 5\% are considered in this report. The ORs were computed compared to haplotype with the highest estimated population frequency. An additive model was tested adjusting for maternal age, maternal BMI, parity, and family history of diabetes. The false discovery rate (FDR) method with a significance level of 0.2 was applied for multiple comparisons. Gene-by-gene interactions were detected using the method of generalized multivariate dimensionality reduction (GMDR) [14]. 
2.5. Results. The characteristics of the cases and controls have already been described elsewhere [11]. In brief, there were more GDM cases with a higher BMI and family history of diabetes than control subjects $(P=0.0002, P=0.005)$. None of the cases or controls consumed alcohol during pregnancy. The distributions of maternal age, weight gain during pregnancy, parity, high blood pressure during pregnancy, passive smoking, and gestational weeks between cases and controls were similar.

All associations between genotypes in the seven genes and GDM risk are shown in Supplementary Table 2. Significant associations with GDM risk in the CDKAL1 gene are shown in Table 1; risk alleles of $A, G$, and $G$ in rs9295478, rs6935599, and rs7747752 were found to be associated with elevated GDM risk in additive, dominant, codominant, and recessive models. These alleles could increase the GDM risk for around 2 times in the additive model, 1.45 times in the dominant model, 1.4 times in the codominant model, and 1.8 times in the recessive model. These associations remained statistically significant after adjusting for multiple comparisons in additive, recessive, and codominant models for rs9295478 and rs6935599.

$G$ alleles in rs4712527, rs7748720, rs9350276, and rs6938256 were found to be associated with reduced GDM risk compared with A alleles in all four models as mentioned above. GDM risks in women who carried G alleles in rs4712527 and rs7748720 were approximately 0.20 times in the additive model, 0.50 times in dominant and codominant models, and 0.20 times in the recessive model compared with women who carried A alleles. GDM risks in women who carried G alleles in rs9350276 and rs6938256 were around 0.40 times in the additive model, 0.60 times in dominant and codominant models, and 0.50 times in the recessive model compared with women who carried A alleles. After adjusting for multiple comparisons, the associations for rs7748720 and rs6938256 remained significant in dominant and codominant models, and rs4712527 remained significant in the codominant model; however, the nonsignificant results were found in rs9350276.

As shown in Table 2, CDKAL1 gene polymorphisms were significantly associated with risk of GDM at the gene level, and this gene was still statistically significant after adjusting for multiple comparisons. Nonsignificant results were found in IRS1, IGF2BP2, GCK, TCF7L2, KCNQ1, and KCNJ11 genes.

We observed strong linkage disequilibrium of 16 blocks in 111 SNPs in the CDKAL1 gene (Figure 1). SNPs significantly associated with GDM that are listed in Table 1 in the manuscript including negative SNPs (rs4712527 and rs7748720) and positive SNPs (rs9295478, rs6935599, and rs7747752) belong to the same block 4, another SNP rs9350276 belong to block 5, and rs6938256 does not belong to any block constructed by HaploView. The GACGGACG haplotype in block 4 and the AG haplotype in block 5 were associated with reduced risk of GDM. Haplotype analyses were consistent with the results of the individual SNP analyses and did not provide additional insight into these associations (Table 3). Gene and gene interaction analysis using GMDR did not find significant results.

\section{Discussion}

We verified associations between polymorphisms in genes including TCF7L2, GCK, KCNJ11, KCNQ1, CDKAL1, $I G F 2 B P 2$, and IRS1 and risk of GDM in a Chinese population. At the gene level, CDKAL1 was significantly associated with GDM risk. The CDKAL1 gene located in the short arm of human chromosome 6 could affect the function of $\beta$-cells through inhibiting the activity of $C D K 5$ and acting as a tRNA-modifying enzyme [15], and risk alleles in this gene could affect the process of proinsulin conversion to insulin through protein translation. In our study, several SNPs in the CDKAL1 gene were found to be associated with GDM risk. Among these SNPs, the G allele in rs4712527 was reported to be associated with reduced risk of T2DM in a cohort study from Argentina [16], but not in a Chinese population [17]. CDKAL1 gene polymorphisms including rs7754840 and rs10946398 were reported to be associated with risk of T2DM in Asian populations [18, 19]. Results from a GWA study in Korean women suggested that rs7754840 in CDKAL1 is strongly associated with GDM [20], and subsequent meta-analysis also confirmed that the $\mathrm{C}$ allele of rs7754840 was associated with elevated risk of GDM [6]. A study from India did not find a significant association between CDKAL1 polymorphisms and GDM risk [21]. Another study from Russia examined whether CDKAL1 polymorphisms modified the relationship between lifestyles including food consumption, physical activity, and smoking as well as GDM risk and it did not find significant interactions; however, results from this study suggest that the association of sausage consumption with GDM risk can be determined based on the number of risk alleles of rs1799884 in GCK [22]. The CDKAL1 rs10946398 CC genotype was reported to be associated with the need for insulin therapy in a study conducted in Poland; however, the association did not pass the statistical significance threshold after correction for multiple testing [23]. Rs7748720 in CDKAL1 was found to be significantly associated with GDM risk; however, it was never reported in previous studies, and it still has to be verified by further studies in a different population.

Although the precise pathology of GDM remains unclear, it was reported to be related to increased insulin resistance and/or decreased insulin sensitivity during pregnancy [1]. Therefore, the association between GDM risk and CDKAL1 polymorphisms could be supported by its relation to these metabolic characteristics. In a Cardiometabolic Risk in Chinese (CRC) study, rs 10946398 of CDKAL1 was associated with markers of impaired insulin secretion, suggesting that its effect on glucose-related traits might have a role in the development of GDM [24]. Among Greek children, rs9356744 and rs2206734 were related to insulin resistance, a clinical marker related to T2DM in a cross-sectional cohort of Greek children and adolescents of European descent [25]. In a meta-analysis, rs9460557 of CDKAL1 showed evidence with insulin clearance and suggested it may have a 


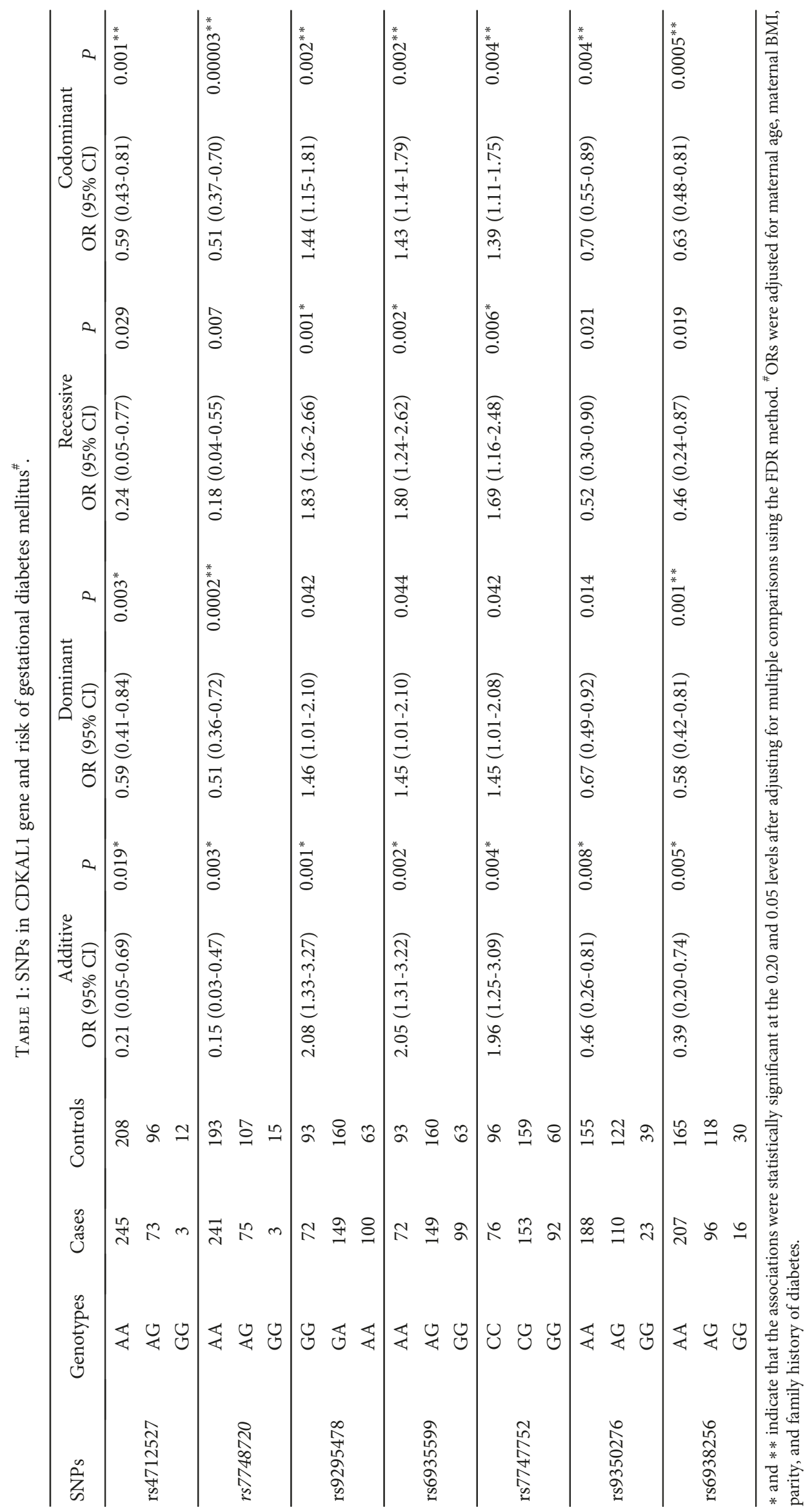


TABLE 2: Gene polymorphisms and risk of gestational diabetes mellitus.

\begin{tabular}{|c|c|c|c|c|c|c|}
\hline Genes & Location & Start & End & Number of SNPs & $\min P$ & $\min P^{*}$ \\
\hline IRS1 & $2 \mathrm{q} 36.3$ & $227,596,033$ & $227,664,485$ & 4 & 0.362 & 0.656 \\
\hline IGF2BP2 & $3 q 27.2$ & $185,361,527$ & $185,542,844$ & 14 & 0.515 & 0.709 \\
\hline CDKAL1 & $6 \mathrm{p} 22.3$ & $20,534,688$ & $21,232,635$ & 111 & 0.001 & 0.007 \\
\hline GCK & $7 \mathrm{p} 13$ & $44,183,870$ & $44,237,769$ & 15 & 0.157 & 0.550 \\
\hline TCF7L2 & $10 \mathrm{q} 25.2$ & $114,710,009$ & $114,927,437$ & 43 & 0.608 & 0.709 \\
\hline KCNQ1 & $11 \mathrm{p} 15.5$ & $2,465,914$ & $2,870,340$ & 107 & 0.375 & 0.656 \\
\hline KCNJ11 & $11 \mathrm{p} 15.1$ & $17,406,795$ & $17,410,878$ & 1 & 0.923 & 0.923 \\
\hline
\end{tabular}

$\min P^{*}$ after adjusting for multiple comparisons using the method of FDR.

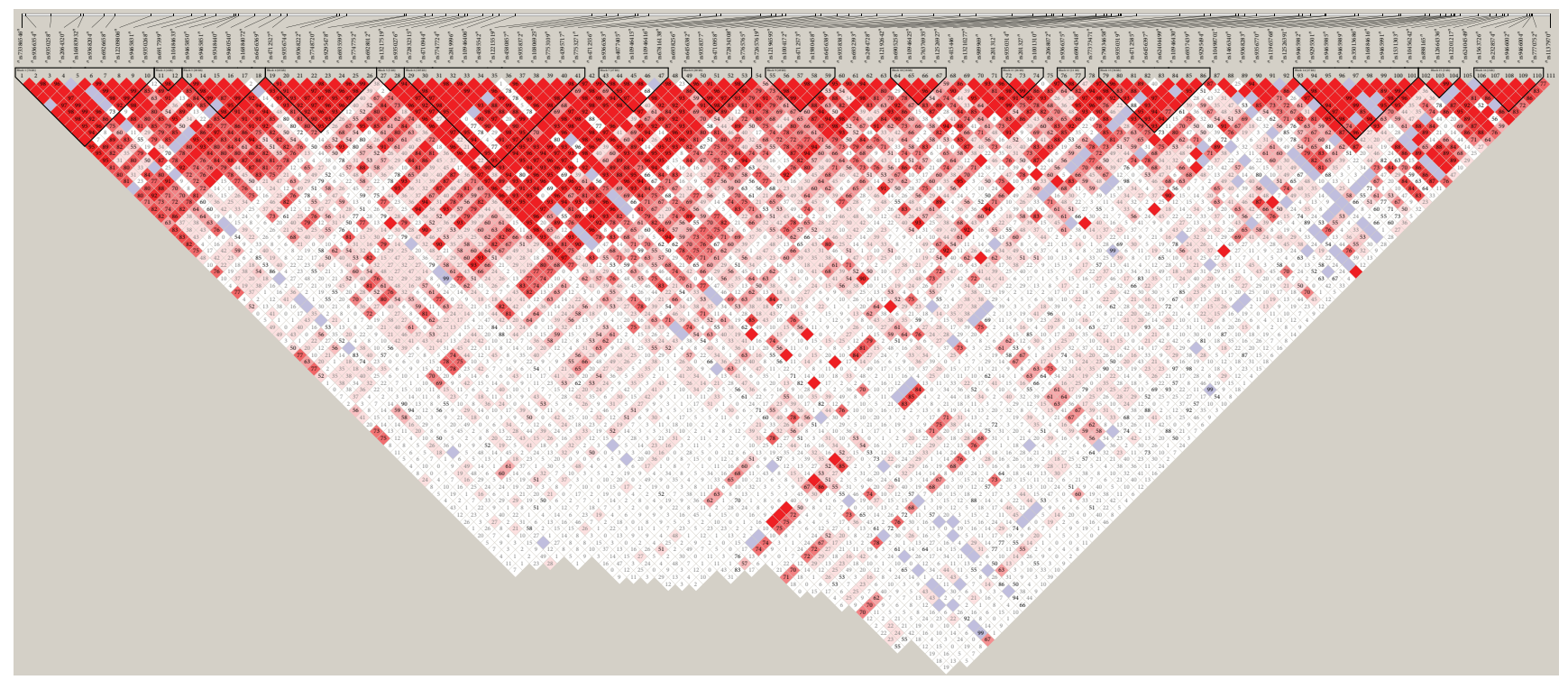

Figure 1: LD plot of 111 SNPs in the CDKAL1 gene.

TABle 3: Association of haplotypes in the CDKAL1 gene with gestational diabetes mellitus.

\begin{tabular}{lcccc}
\hline SNP combinations & Frequency (\%) & $\mathrm{OR}^{\mathrm{a}}$ & $95 \% \mathrm{CI}$ & $P^{\mathrm{b}}$ \\
\hline Block 4 & & & & \\
rs4712527 rs9356744 rs9368222 rs7748720 rs9295478 rs6935599 \\
rs7747752 rs6928012 \\
$\quad$ & 39.88 & 1 & & \\
$\quad$ AGAAAGGG & 31.24 & 0.82 & $0.62-1.07$ & 0.145 \\
AACAGACA & 14.08 & 0.52 & $0.37-0.74$ & 0.0003 \\
GACGGACG & 6.72 & 0.98 & $0.62-1.55$ & 0.931 \\
AACAAGGG & 8.08 & 0.86 & $0.56-1.33$ & 0.505 \\
Rare (<0.05) & & & & \\
\hline Block 5 & & & & \\
rs13217519 rs9350276 & 36.73 & 1 & & \\
$\quad$ GA & 35.32 & 1.03 & $0.79-1.33$ & 0.845 \\
AA & 27.94 & 0.71 & $0.54-0.93$ & 0.015 \\
AG & & & &
\end{tabular}

pleiotropic effect on insulin secretion, insulin sensitivity, and insulin clearance [26]. rs7756992 was also validated in relation to these intermediate traits related to T2DM in an Indian population [27].

GWA and candidate gene studies have increased our knowledge of loci associated with diabetes; however, applying these findings to infer pathophysiology and promote drug discovery remains challenging. CDKAL1 was revealed to be an inhibitor of $C D K 5$ to suppress the $\beta$-cell differentiation and further highlighted its importance in diabetes [28]. T2DM-related alleles of SNPs located within the CDKAL1 gene region were reported to be associated with impaired $\beta$-cell function [29] and impaired insulin secretion $[24,30]$, which may suggest the role of CDKAL1 in the development of GDM. Mutation in CDKAL1 led to impaired glucose secretion in vitro and vivo [31], and a high-content chemical screen identified a candidate drug that rescued CDKAL1specific defects by inhibiting the FOS/JUN pathway [31]. This may help to the precise therapy of diabetes.

Strengths and limitations should be considered when interpreting the study results. Firstly, diagnosis of GDM in our study was acquired by investigating the well- 
documented medical records, which minimized potential disease misclassification. Secondly, information on potential confounders was collected using a standardized and structured questionnaire allowing for the control of potential confounding effects. The current study was hospital-based, which might have limited the generalizability of these study results. Thirdly, the BMI was not equally distributed between cases and control subjects; however, it was adjusted in each model analyzed. The results in our study will need to be verified by larger population-based studies. Lastly, the sample size in our study was moderate, and the weak effects of SNPs on GDM may not have been observed.

To the best of our knowledge, our study for the first time found a novel association between rs7748720 of CDKAL1 and GDM risk. This would help increase our knowledge of loci associated with GDM and infer the pathophysiology; however, additional studies still need to verify the results.

\section{Data Availability}

The data used to support the findings of this study are included within the article and supplementary information files.

\section{Ethical Approval}

All study procedures were approved by the Human Investigation Committee at the Shanxi Medical University.

\section{Consent}

Informed consent to inclusion in the study was obtained from all individual participants included in the study.

\section{Conflicts of Interest}

The authors declare that they have no conflict of interest.

\section{Authors' Contributions}

Keke Wang, Qiong Chen, Suping Wang, and Yawei Zhang contributed equally to this article.

\section{Acknowledgments}

This work was supported by the National Natural Science Foundation of China grants (No. 81473061), Natural Science Foundation of Shanxi Province grants (No. 2013021033-2), "100 Talent Plan" Award of Shanxi Province, Construction Project of Characteristic Key Disciplines from Universities of Shanxi Province, and "10 Talent Plan" Award of Shanxi Medical University. The authors express their appreciation to the participants in the Taiyuan Birth Cohort Study for their enthusiastic support.

\section{Supplementary Materials}

Supplementary Table 1: genes and SNPs evaluated. Supplementary Table 2: genotypes and risk of gestational diabetes mellitus. (Supplementary Materials)

\section{References}

[1] E. Chiefari, B. Arcidiacono, D. Foti, and A. Brunetti, "Gestational diabetes mellitus: an updated overview," Journal of Endocrinological Investigation, vol. 40, no. 9, pp. 899909, 2017.

[2] Y. Zhu and C. Zhang, "Prevalence of gestational diabetes and risk of progression to type 2 diabetes: a global perspective," Current Diabetes Reports, vol. 16, no. 1, p. 7, 2016.

[3] A. Ferrara, "Increasing prevalence of gestational diabetes mellitus: a public health perspective," Diabetes Care, vol. 30, Supplement 2, pp. S141-S146, 2007.

[4] A. Ben-Haroush, Y. Yogev, and M. Hod, "Epidemiology of gestational diabetes mellitus and its association with type 2 diabetes," Diabetic Medicine, vol. 21, no. 2, pp. 103-113, 2004.

[5] W. L. Lowe, D. M. Scholtens, V. Sandler, and M. G. Hayes, "Genetics of gestational diabetes mellitus and maternal metabolism," Current Diabetes Reports, vol. 16, no. 2, p. 15, 2016.

[6] C. Zhang, W. Bao, Y. Rong et al., "Genetic variants and the risk of gestational diabetes mellitus: a systematic review," Human Reproduction Update, vol. 19, no. 4, pp. 376-390, 2013.

[7] J. Robitaille and A. M. Grant, "The genetics of gestational diabetes mellitus: evidence for relationship with type 2 diabetes mellitus," Genetics in Medicine, vol. 10, no. 4, pp. 240 250, 2008.

[8] V. Radha, S. Kanthimathi, R. M. Anjana, and V. Mohan, "Genetics of gestational diabetes mellitus," The Journal of the Pakistan Medical Association, vol. 66, no. 9, pp. S11-S14, 2016.

[9] A. Stuebe, A. Wise, T. Nguyen, A. Herring, K. North, and A. Siega-Riz, "Maternal genotype and gestational diabetes," American Journal of Perinatology, vol. 31, no. 1, pp. 6976, 2014.

[10] A. Huerta-Chagoya, P. Vazquez-Cardenas, H. Moreno-Macias et al., "Genetic determinants for gestational diabetes mellitus and related metabolic traits in Mexican women," PLoS One, vol. 10, no. 5, article e0126408, 2015.

[11] Q. Chen, H. Yang, Y. Feng et al., "SOS1 gene polymorphisms are associated with gestational diabetes mellitus in a Chinese population: results from a nested case-control study in Taiyuan, China," Diabetes and Vascular Disease Research, vol. 15, no. 2, pp. 158-161, 2018.

[12] C. Ober, K. S. Xiang, R. A. Thisted et al., "Increased risk for gestational diabetes mellitus associated with insulin receptor and insulin-like growth factor II restriction fragment length polymorphisms," Genetic Epidemiology, vol. 6, no. 5, pp. 559-569, 1989.

[13] E. C. Tok, D. Ertunc, O. Bilgin, E. M. Erdal, M. Kaplanoglu, and S. Dilek, "Association of insulin receptor substrate-1 G972R variant with baseline characteristics of the patients with gestational diabetes mellitus," American Journal of Obstetrics and Gynecology, vol. 194, no. 3, pp. 868-872, 2006.

[14] N. Shaat, M. Ekelund, A. Lernmark et al., "Association of the E23K polymorphism in the KCNJ11 gene with gestational diabetes mellitus," Diabetologia, vol. 48, no. 12, pp. 25442551, 2005.

[15] C. J. Palmer, R. J. Bruckner, J. A. Paulo et al., "Cdkal1, a type 2 diabetes susceptibility gene, regulates mitochondrial function in adipose tissue," Molecular Metabolism, vol. 6, no. 10, pp. 1212-1225, 2017.

[16] J. D. L. Pinto, P. Yang, A. Sein et al., “The rs4712527 polymorphism in the CDKAL1 gene, as a protective predictor of 
proliferative diabetic retinopathy development in type 2 diabetic patients," Investigative Ophthalmology \& Visual Science, vol. 58, no. 8, p. 1848, 2017.

[17] L. L. Fu, Y. Lin, Z. L. Yang, and Y. B. Yin, "Association analysis of genetic polymorphisms of TCF7L2, CDKAL1, SLC30A8, HHEX genes and microvascular complications of type 2 diabetes mellitus," Zhonghua yi xue yi Chuan xue za zhi= Zhonghua Yixue Yichuanxue Zazhi = Chinese Journal of Medical Genetics, vol. 29, no. 2, pp. 194-199, 2012.

[18] Y. Mansoori, A. Daraei, M. M. Naghizadeh, and R. Salehi, "Significance of a common variant in the CDKAL1 gene with susceptibility to type 2 diabetes mellitus in Iranian population," Advanced Biomedical Research, vol. 4, no. 1, p. $45,2015$.

[19] Y. C. Chang, P. H. Liu, Y. H. Yu et al., "Validation of type 2 diabetes risk variants identified by genome-wide association studies in Han Chinese population: a replication study and meta-analysis," PLoS One, vol. 9, no. 4, article e95045, 2014.

[20] S. H. Kwak, S. H. Kim, Y. M. Cho et al., "A genome-wide association study of gestational diabetes mellitus in Korean women," Diabetes, vol. 61, no. 2, pp. 531-541, 2012.

[21] A. E. Noury, O. Azmy, J. Alsharnoubi, S. Salama, A. Okasha, and W. Gouda, "Variants of CDKAL1 rs7754840 (G/C) and CDKN2A/2B rs10811661 (C/T) with gestational diabetes: insignificant association," BMC Research Notes, vol. 11, no. 1, p. 181, 2018.

[22] P. V. Popova, A. A. Klyushina, L. B. Vasilyeva et al., "Effect of gene-lifestyle interaction on gestational diabetes risk," Oncotarget, vol. 8, no. 67, pp. 112024-112035, 2017.

[23] M. Tarnowski, D. Malinowski, K. Pawlak, V. Dziedziejko, K. Safranow, and A. Pawlik, "GCK, GCKR, FADS1, DGKB/ TMEM195 and CDKAL1 gene polymorphisms in women with gestational diabetes," Canadian Journal of Diabetes, vol. 41, no. 4, pp. 372-379, 2017.

[24] J. Liang, Y. Pei, X. Liu et al., “The CDKAL1 gene is associated with impaired insulin secretion and glucose-related traits: the Cardiometabolic Risk in Chinese (CRC) study," Clinical Endocrinology, vol. 83, no. 5, pp. 651-655, 2015.

[25] M. Rask-Andersen, G. Philippot, G. Moschonis et al., "CDKAL1-related single nucleotide polymorphisms are associated with insulin resistance in a cross-sectional cohort of Greek children," PLoS One, vol. 9, no. 4, article e93193, 2014.

[26] M. O. Goodarzi, X. Guo, J. Cui et al., "Systematic evaluation of validated type 2 diabetes and glycaemic trait loci for association with insulin clearance," Diabetologia, vol. 56, no. 6, pp. 1282-1290, 2013.

[27] V. Gupta, D. G. Vinay, S. Rafiq et al., "Association analysis of 31 common polymorphisms with type 2 diabetes and its related traits in Indian sib pairs," Diabetologia, vol. 55, no. 2, pp. 349-357, 2012.

[28] K. C. Liu, G. Leuckx, D. Sakano et al., "Inhibition of Cdk5 promotes $\beta$-cell differentiation from ductal progenitors," Diabetes, vol. 67, no. 1, pp. 58-70, 2017.

[29] L. Pascoe, A. Tura, S. K. Patel et al., "Common variants of the novel type 2 diabetes genes CDKAL1 and HHEX/IDE are associated with decreased pancreatic $\beta$-cell function," Diabetes, vol. 56, no. 12, pp. 3101-3104, 2007.

[30] A. Stancáková, J. Pihlajamäki, J. Kuusisto et al., "Singlenucleotide polymorphism rs7754840 of CDKAL1 is associated with impaired insulin secretion in nondiabetic offspring of type 2 diabetic subjects and in a large sample of men with normal glucose tolerance," The Journal of Clinical Endocrinology \& Metabolism, vol. 93, no. 5, pp. 1924-1930, 2008.

[31] H. Zeng, M. Guo, T. Zhou et al., "An isogenic human ESC platform for functional evaluation of genome-wide-associationstudy-identified diabetes genes and drug discovery," Cell Stem Cell, vol. 19, no. 3, pp. 326-340, 2016. 


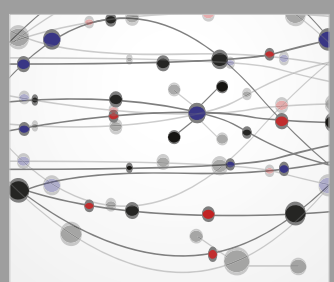

The Scientific World Journal
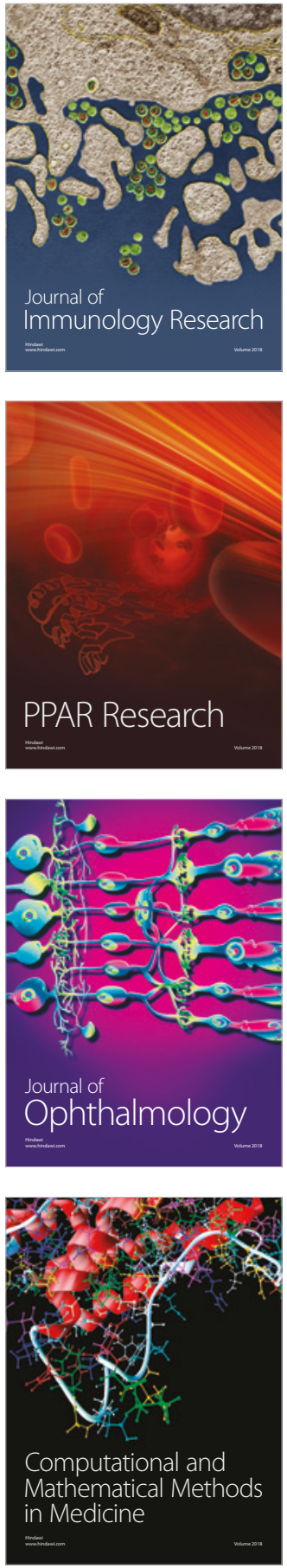

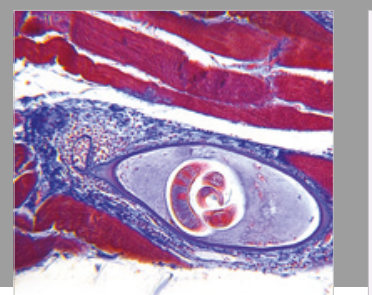

Gastroenterology Research and Practice

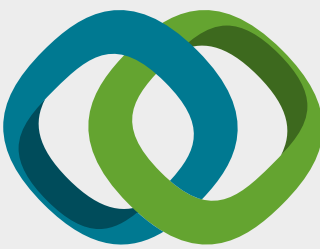

\section{Hindawi}

Submit your manuscripts at

www.hindawi.com
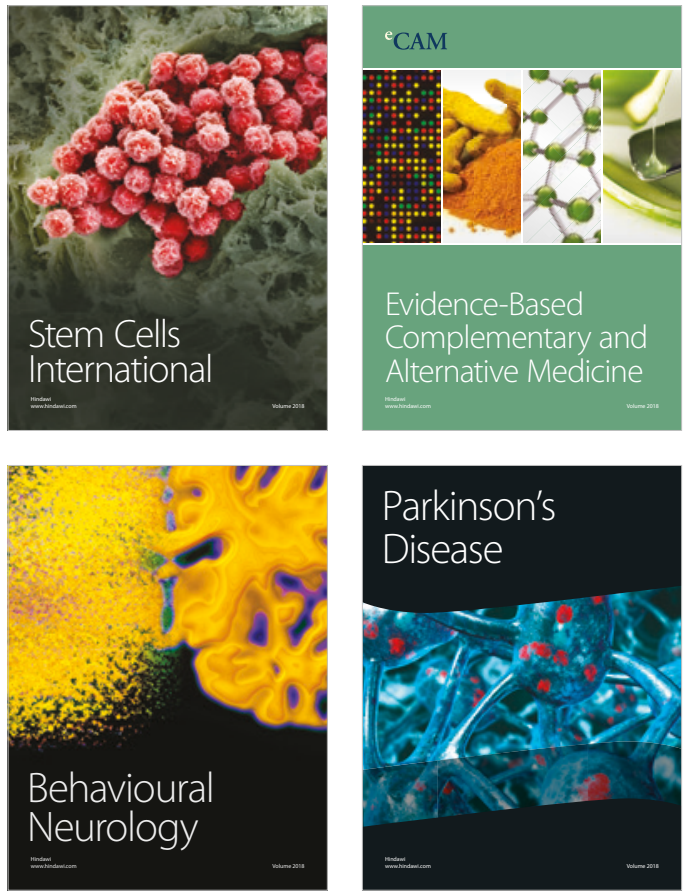

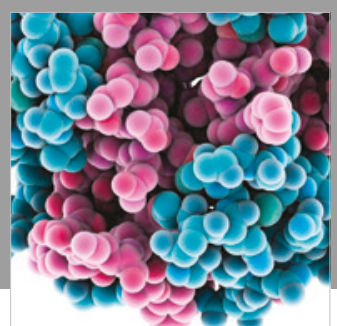

ournal of

Diabetes Research

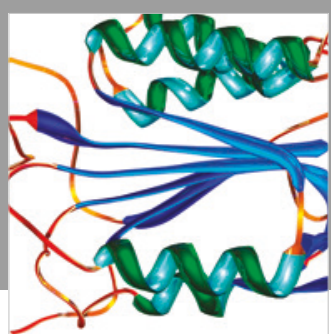

Disease Markers
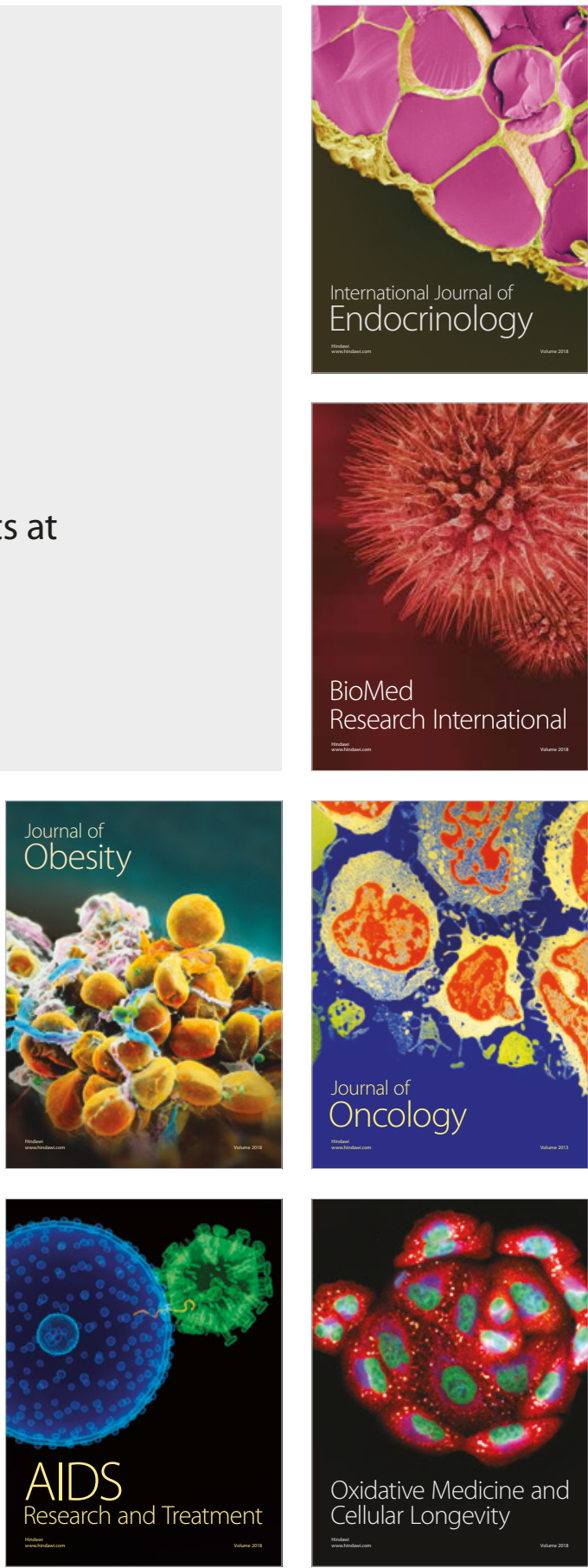